\title{
Parallel, divers, nachhaltig, anders: Wie Tales from the Town of Widows (2007) dazu beiträgt, Gesellschaft neu zu denken
}

\begin{abstract}
The day will come when men will recognize woman as his peer, not only at the fireside, but in councils of the nation. Then, and not until then, will there be the perfect comradeship, the ideal union between the sexes that shall result in the highest development of the race.

(Anthony, zit. in Cañón 2007, n. pag.)
\end{abstract}

Mit diesem bekannten Zitat der US-amerikanischen Frauenrechtlerin und Schriftstellerin Susan Brownell Anthony, welches ihrer Publikation The Status of Women, Past, Present, and Future von 1897 entnommen ist, leitet der kolumbianische, aber in den Vereinigten Staaten von Amerika ansässige Autor James Cañón seinen 2007 erschienenen Debütroman Tales from the Town of Widows ein. Indem er es über hundert Jahre später als Prolog zu seiner literarischen Utopie verwendet, bekräftigt Cañón nicht nur die Ideen und Argumente (feministischer) Aktivistinnen der sogenannten Progressive Era, sondern zeigt auch kritisch auf, dass sich Anthonys im späten neunzehnten Jahrhundert artikulierte Vorhersage zur gesellschaftlichen Gleichstellung und Diversität noch nicht realisiert hat. Indem er nach wie vor virulente soziale Spannungen in puncto Patriarchat und Genderdebatte widerspiegelt, diese kritisch hinterfragt und sogar ein alternatives Gesellschaftsmodell aufzeigt, liefert der Roman einen Anstoß, um Gesellschaft neu zu denken. Dabei soll die vorliegende Analyse des Romans die Richtung einschlagen, die Teresa Hiergeist in ihrer Einleitung zum Sammelband Parallel- und Alternativgesellschaften in den Gegenwartsliteraturen formuliert. Das Bestreben dieser Publikation ist es, sich eines Desiderats in der Literaturwissenschaft anzunehmen und auch in diesem akademischen Feld Forschungsgegenstände für die Debatte um ,paragesellschaftliche` Phänomene fruchtbar machen. Eine solche Herangehensweise rückt in den Fokus, inwiefern Literatur „Fundamente des Zusammenlebens“ neu diskutiert und ,in der Konstruktion [einer] utopische[n] Gesellschaft[...] eigene alternative Sozialitätsvorschläge“ (2017, 9) entwirft. Dieser Beitrag zu Tales from the Town of Widows soll also aufzeigen, inwiefern der Roman in seiner fiktionalen Darstellung einer neu entstehenden diversen Gemeinschaft dazu beiträgt, Gesellschaft neu zu konzipieren.

Ә Open Access. (C 2021 Claudia Hachenberger, publiziert von De Gruyter. (c) BY-NC-ND Dieses Werk ist lizenziert unter einer Creative Commons Namensnennung - Nicht-kommerziell - Keine Bearbeitung 4.0 International Lizenz. https://doi.org/10.1515/9783110707489-011 
Der thematische Fokus des utopischen Szenarios liegt auf der von zahlreichen Bürgerkriegen gepeinigten und machistischen ${ }^{1}$ Gesellschaft Kolumbiens. In seinem Beitrag für The Novelist's Lexicon erzählt Cañón von seinem Anliegen, auf fiktionaler Ebene ein alternatives Gesellschaftsmodell zu entwerfen, welches Diversität sowie eine Gleichstellung der Geschlechter lebt $(2011,65)$. Die imaginäre ,Paragesellschaft' in Tales from the Town of Widows durchbricht binäre Genderoppositionen und hebt sich somit deutlich vom patriarchalisch geprägten Kolumbien ab. Wie Françoise Aubès eindrücklich darlegt, ermöglichten in erster Linie eine räumliche und sprachliche Distanz $(2010,28),{ }^{2}$ dass Cañón seine Vorstellung eines nachhaltigen und diversen alternativen Gesellschaftsmodells, welches gleichzeitig implizit einige politische, soziale und gesellschaftliche Ansätze seines Heimatlandes auf fiktionaler Ebene kritisiert, formulieren konnte. Eine Analyse des zeitgenössischen Romans im Hinblick auf seine Repräsentation einer ,Paragesellschaft" als Alternative für die negativ bewerteten realpolitischen und sozialen Umstände Kolumbiens ist außerordentlich fruchtbar, da er gesellschaftliches Zusammenleben aus einer nicht-eurozentrischen, diversen Perspektive heraus interpretiert und im Gegensatz zu traditionellen Geschlechterutopien ${ }^{3}$ zeigt, dass eine bloße Geschlechtertrennung keine endgültige Lösung für das Problem des Patriarchats bieten kann (Relf 1991, 131).

1 Nicht zuletzt aufgrund der immer noch einflussreichen Rolle der Institution Kirche, welche patriarchalische Wertevorstellungen und Verhaltensweisen autorisiert und aufrecht erhält, sind lateinamerikanische Gesellschaftsmodelle nach wie vor durch einen tiefgreifenden kulturellen Machismo gekennzeichnet, der den heterosexuellen Mann im Vergleich zur Frau als hierarchisch höher gestellt versteht und legitimiert, dass diese (verbal) unterdrückt und häufig Opfer gewalttätiger Übergriffe wird (Quiñones Mayo und Resnick 1996, 263).

2 Bezugnehmend auf Cañóns Wohnsitz in den Vereinigten Staaten (räumliche Trennung) und die Veröffentlichung des Romans auf Englisch statt auf Spanisch (sprachliche Trennung). Die Wahl der Publikationssprache trägt dazu bei, dass sich die Zahl der möglichen Leser^innen weltweit und insbesondere in den Vereinigten Staaten erhöht. Dieser strategische, auch vom hemisphärischen Gedanken geprägte Schritt Cañóns unterstreicht, was Isaac Goldemberg als „americanidad no unidimensional sino multifacética“ [nicht eindimensionaler, sondern facettenreicher Amerikanismus] (zit. in Aubès 2010, 28) bezeichnet. Trotzdem bleibt die fiktionale Erzählung einigen wichtigen Merkmalen des lateinamerikanischen Romans treu, einschließlich der Verwendung magisch-realistischer Elemente und eines Márquez'schen Humors (Aubès 2010, 27).

3 Traditionelle Geschlechterutopien sind durchaus gesellschaftskritisch angelegt, vertiefen jedoch in ihrer fiktionalen Darstellung von rein weiblichen bzw. rein männlichen Gesellschaften mit mitunter interessanten Methoden zur Fortpflanzung, die das Fortbestehen der Gemeinschaften garantieren sollen - die strukturell angelegten und auch institutionell tradierten binären Genderoppositionen. Auf literarischer Ebene verschweigen und übergehen sie dabei die Existenz der ohnehin sozial stigmatisierten und gesellschaftlich marginalisierten LGBTQ. 
Tales from the Town of Widows kann in erster Linie aufgrund seines Inhalts als literarische Utopie kategorisiert werden, vor allem aber auch deswegen, da es den utopischen Diskurs nutzt, um die bestehende politische und patriarchalische Ordnung zu kritisieren und ein alternatives Gesellschaftmodell zu präsentieren. Im folgenden Beitrag soll beleuchtet werden, inwiefern die fiktiven Charaktere der utopischen Erzählung aus einem Aspekt der Unfreiwilligkeit heraus eine räumlich isolierte Gemeinschaft gründen, die sich - in ihrer Struktur und in ihrer Auffassung vom Konvivialismus - gesellschaftskritisch zu tradierten und von der katholischen Kirche untermauerten patriarchalen Grundsätzen positioniert. Die fiktionale Darstellung der friedlichen, divers zusammenlebenden Gruppe, die sowohl lokal als auch institutionell von der ,Mehrheitsgesellschaft‘ abgegrenzt besteht (Biersack et al. 2019, 14), wird dabei als ,Paragesellschaft' verstanden, welche auch auf sozialer Ebene Raum zur Verhandlung bietet (Hiergeist 2017, 8) und Leser ${ }^{\star}$ innen dazu anregt, eigene Verhaltensweisen und Wertevorstellungen zu reflektieren. In den folgenden Abschnitten soll zunächst diskutiert werden, warum der Roman als zeitgenössische und diverse Utopie gelten kann. Weiterhin soll die Darstellung der entstehenden (sozialistisch-autarken) ,Paragesellschaft' in Tales from the Town of Widows nachvollzogen werden. In einem weiteren Unterkapitel erfolgt dann eine Lektüre mittels ,paragesellschaftlicher' Kategorien. Diese soll aufzeigen, inwiefern der Roman es in seiner Schilderung eines Zusammenlebens, welches einen nachhaltigen Umgang mit der Natur, Diversität sowie individuelle Selbstbestimmung priorisiert, vermag, Gesellschaft neu zu denken.

\section{Tales from the Town of Widows als zeitgenössische, diverse Utopie}

Spätestens mit dem Erscheinen von Charlotte Perkins Gilmans Roman Herland im Jahre 1915, in dem die weiblichen Einwohnerinnen von Herland die patriarchale Unterdrückung durch die Auslöschung des männlichen Geschlechts überwunden haben, versuchen feministische Utopien auf fiktionaler Ebene alternative Wege des friedlichen gesellschaftlichen und gleichgeschlechtlichen Zusammenlebens zu entwerfen (Pfaelzer 1984, 151). Im Gegensatz zu traditionellen männlichen Utopien, die weibliche Charaktere in der Regel margi- 
nalisieren und einen individualistischen männlichen Helden präsentieren, ${ }^{4}$ stellen feministische Utopien traditionelle Geschlechterbilder in Frage (Layh 2014, 67-71), da die patriarchalische Herrschaft hier zunehmend als unerträglich und unhaltbar empfunden wird (Werder 2009, 157). Obwohl es auf den ersten Blick kontrovers erscheinen mag, im Falle von Cañóns Roman von einer feministischen Utopie zu sprechen, da solche Texte in erster Linie deshalb als feministisch bezeichnet werden, da sie von Autorinnen verfasst wurden (Klarer 1993, 1-7), möchte ich mit einer solchen binären und rein autororientieren Kategorisierung brechen. In einem ersten Schritt ordne ich Cañóns Roman daher in die Kategorie der feministischen Utopie ein, weil er sich - wie Herland - klar von patriarchalischen Stereotypen distanziert (Klarer 1993, 48). In einem zweiten Schritt möchte ich zeigen, dass der Roman in seiner Thematisierung von Ungerechtigkeit noch weiter geht, LGBTQ nicht unerwähnt lässt und binäre Genderoppositionen ausdehnt, indem er eine diverse und nachhaltige ,Paragesellschaft als alternatives Gesellschaftsmodell anbietet und somit aktiv dazu beiträgt, Gesellschaft neu zu denken.

In Anlehnung an das narrative Schema gleichgeschlechtlicher Utopien, in der das ,andere“ Geschlecht durch eine Naturkatastrophe oder einen Krieg ausgelöscht wird, wählt Cañón für seinen Roman einen ähnlichen Ausgangspunkt. Wie der heterodiegetische Erzähler schildert, wird das friedliche patriarchalische Dorf Mariquita eines Tages von kommunistischen Guerilla-Kämpfern überfallen. ${ }^{5}$ Dabei ermorden oder entführen die guerrilleros fast alle männlichen Einwohner. Zurück bleiben neben den traumatisierten Dorfbewohnerinnen nur der moralisch und ethisch korrumpierte katholische Priester, eine Transfrau und

\footnotetext{
4 Im Allgemeinen sind die Gesellschaften, die in traditionellen männlichen Utopien dargestellt werden, nicht gleichgeschlechtlich. Weibliche Charaktere sind Teil männlicher Utopien, allerdings halten die Gesellschaften an patriarchalen und damit stereotypisierenden Geschlechterrollen fest; die Frage nach der Gleichberechtigung wird totgeschwiegen oder bestenfalls marginalisiert (Schölderle 2011, 306). Gleichgeschlechtliche männliche Utopien sind weniger häufig als feministische Utopien. Da sich Patriarchen in der Regel nicht gesellschaftlich unterdrückt oder benachteiligt fühlen, würde die Vorstellung einer Welt ohne Frauen nicht automatisch einen Zuwachs an Freiheit oder Selbstbestimmung bedeuten, weshalb sie nicht als besonders reizvoll angesehen wird. Im 20. Jahrhundert unterstreichen fiktionale Texte, die rein männliche Gesellschaften konzipieren, entweder religiöse Werte (schlechter Einfluss von Frauen) oder stellen Werte des Militarismus in den Vordergrund (Romaine 1999, 329).

5 Im Roman wird das Ereignis, welches das Leben der Dorfbewohner so gewaltsam unterbricht, wie folgt beschrieben: „Immediately after the ninth stroke of the church bell, while its echo was still resounding in the sexton's ears, three dozen men in worn-out greenish uniforms appeared from every corner of Mariquita shooting their rifles and shouting, Viva la Revolución!“ (Cañón 2007, 3).
} 
ein Homosexueller, der ein zurückgezogenes und eher einsames Leben führt. ${ }^{6}$ Unfreiwillig vor die folgenschwere Situation gestellt, ihrer Ehemänner, Liebhaber oder Söhne beraubt worden zu sein, sehen die selbsternannten ,Witwen ${ }^{6}$ nach der erfolgreichen Bewältigung zahlreicher Hindernisse eine Chance, sich endlich von patriarchalischen Zwängen und ihrer inferioren Stellung im traditionellen Rollengefüge $\mathrm{zu}$ lösen, um selbstbestimmte Identitäten innerhalb einer ökologischorientierten und diversen ,Paragesellschaft' ${ }^{\star} \mathrm{zu}$ entwickeln. Dabei ist es für die Gründung der alternativen Gesellschaft von New Mariquita außerordentlich hilfreich, dass eine räumliche Abgrenzung zum Rest Kolumbiens und somit zur dort immer noch vorherrschenden patriarchalisch geprägten ,Mehrheitsgesellschaft vorgenommen wird. Der Roman ist nicht als Zeitutopie, sondern als Raumutopie angelegt. Dabei wird die diverse Gemeinschaft von New Mariquita eben nicht als Zukunftsvision angesehen, es ist vielmehr die Isolation, die ein Umdenken der Charaktere und eine Abspaltung begünstigt: Eines Tages verwüstet ein Sturm alle Zufahrtsstraßen zum Dorf (Cañón 2007, 147) und der schnell zuwachsende Dschungel als undurchdringliche natürliche Barriere (Cañón 2007, 227) tut sein Übriges, so dass das Dorf nicht mehr „on a recently updated map“ (Cañón 2007, 107) lokalisiert werden kann.

Feministische Utopien werden in der Regel als friedlich dargestellt, sind aber in einigen Fällen durch männliche Aggression und Gewalt bedroht. Im Gegensatz zur präsentierten Realität in rein weiblichen Gemeinschaften verurteilen männliche Charaktere diese als kriegerisch und kannibalisch. Nach ihrer Vorstellung werden die weiblichen Gemeinschaften von Frauen bewohnt, die ihnen körperlich überlegen sind, völlig unabhängig von ihnen leben und sogar erfolgreich blutige Kriege gegen patriarchale und männerdominierte Gemeinschaften führen (Klarer 1993, 11-12). Die Figur des Reisenden, der sich aus der ,Mehrheitsgesellschaft‘ heraus auf den Weg macht, um die alternative Gesellschaft $\mathrm{zu}$ finden und über sie $\mathrm{zu}$ berichten, wird in Tales from the Town of Widows vom US-amerikanischen Reporter Gordon Smith verkörpert. Im Vorfeld seiner Reise erfährt er von einem „tribe of ferocious female warriors living in a small village deep in the cordillera" und

6 Offensichtlich werden die drei Charaktere (zunächst) nicht als patriarchalische Bedrohung oder Gefahr für die sich im Aufbau befindende alternative Gemeinschaft empfunden. Der nicht-binäre Ansatz des Romans schließt vom Patriarchat benachteiligte Randfiguren ein und teilt die Forderung der Autoren des Convivialist Manifesto, sich bei der Konzeption einer neuen Gesellschaftsform über sozial definierte dichotomische Unterschiede wie Geschlecht oder sexuelle Orientierung hinwegzusetzen $(2014,30)$.

7 Der Begriff ,Witwen“ umfasst die weiblichen Einwohnerinnen von New Mariquita (auch diejenigen, die vor dem Überfall alleinstehend waren) sowie die von den guerrilleros zurückgelassenen LGBTQ des Dorfes. 
von „grotesque, man-hating, heretic, cannibalistic women of gigantic proportions“ (Cañón 2007, 276). Dennoch kämpft sich der neugierige Reporter durch unwegsames Gelände, bis er es schafft, die abgelegene Gemeinde New Mariquita zu erreichen.

In seiner Darstellung einer isolierten Gemeinschaft, die lokal und später auch institutionell abgegrenzt zur ,Mehrheitsgesellschaft‘ besteht, welche wiederum die Bewohner^innen dieser alternativen Gesellschaft als gefährliche Bedrohung sieht und die Figur des Reisenden stellt, der über sie berichten soll, teilt Tales from the Town of Widows also ausgewählte Merkmale feministischer Utopien. Allerdings unterscheidet sich Cañóns Roman auch von traditionellen feministischen Utopien, da er eine dynamische und kritische Vorstellung von Utopie nahbar macht. In seiner fiktionalen Darstellung einer diversen ,Paragesellschaft', die über den rein feministischen Aspekt hinausgeht, nimmt er keine unvoreingenommene Position ein, sondern übt ausdrücklich Kritik an der Geschlechterungerechtigkeit im patriarchalischen System. Die Entwicklung der diversen ,Paragesellschaft‘ wird hierbei als fortlaufender Prozess der Identitätsbildung dargestellt, ein Merkmal, welches für zeitgenössische Utopien als typisch gelten kann (McDowell 2008, 23). Im Folgenden soll nun detaillierter ausgeführt werden, wie dieser dynamische Prozess der schrittweisen Ablösung von der ,Mehrheitsgesellschaft“ im Roman vollzogen wird und wie das entstehende alternative Gesellschaftsmodell, das gleichzeitig das patriarchalisch geprägte Gesellschaftsgefüge Kolumbiens kritisiert, auf fiktionaler Ebene dargestellt wird.

\section{Die Darstellung des entstehenden alternativen Gesellschaftsmodells im Roman}

In den ersten Jahren nach dem Verschwinden der machistischen Dorfbewohner beginnen die Witwen, sich Sorgen um die Zukunft der Ansiedlung zu machen. Sie verspüren ein dringendes Bedürfnis danach, sich fortzupflanzen: „Mariquita's women had to bear boys soon, or else their village would disappear with the present generation“ (Cañón 2007, 147). Die Mehrheit der Frauen wünscht sich, dass der homosexuelle Santiago Marín die Aufgabe übernimmt (Cañón 2007, 147), doch der Priester als Vertreter patriarchalischer Werte ist in der Lage, sie davon zu überzeugen, dass es Gottes Wille ist, dass er sein Keuschheitsgelübde bricht, um Mariquita dabei zu helfen, am Leben zu bleiben (Cañón 2007, 149). Während der durch ihn angestoßenen, jedoch von den Witwen kontrovers diskutierten Fortpflanzungskampagne unter dem biblischen Motto „be fruitful and multiply“ (Cañón 2007, 158) besucht der katholische Priester nach 
und nach die Witwen in ihren Häusern. Nach einer Weile wird jedoch klar, dass er „as sterile as a mule“ (Cañón 2007, 169) ist und mithin lediglich seine Machtposition ausgenutzt hat. Der nächste Versuch der Fortpflanzung wird als „Next Generation Decree“ deklariert, welches besagt, dass die vier Jungen des Dorfes eine der Witwen heiraten müssen, sobald sie ihren fünfzehnten Geburtstag feiern (Cañón 2007, 168-169). Der Priester unterstützt diese Idee der Witwen allerdings nicht, vergiftet die Jungen kurz vor der Trauung im Namen des Herrn (Cañón 2007, 195-196) und vernichtet somit die letzte Hoffnung der Bewohner*innen Mariquitas, auf natürlichem Wege Kinder zu gebären. Nach diesem zweiten Vorfall verspüren diese einen „profound distaste for Catholicism in the[ir] mouths“ (Cañón 2007, 201) und beschließen, den Priester als Vertreter des Patriarchats zu verstoßen. Da ihre Männer sie in Vereinbarkeit mit patriarchalischen Wertevorstellungen misshandelt, ignoriert und diskriminiert haben (Cañón 2007, 170), sind sich die Witwen einig, nicht weiter versuchen $\mathrm{zu}$ wollen, sich fortzupflanzen und dem harmonischen Gefühl der Zusammengehörigkeit in ihrer neuen Gemeinschaft Vorrang einzuräumen: ,[I]f not having men around meant that Mariquita had to end with the present generation, perhaps an entire generation of harmony, tolerance and love would be preferable to an eternity of misery and despair“ (Cañón 2007, 170).

Nach der Vertreibung des Priesters bleibt die Kirchturmuhr stehen, da er den Schlüssel entwendet hat, um diese aufzuziehen (Cañón 2007, 202). Dieser Vorfall verändert das alltägliche Leben der Witwen, da sie fortan von der Tyrannei der Zeit befreit sind (Cañón 2007, 203). Um dem entstehenden Zeitdilemma zu entgehen, entwickeln zwei von ihnen ein neues Zeitkonzept für ihre Gemeinschaft. Dieses basiert auf dem weiblichen Zyklus (Cañón 2007, 219). Mit diesem relationalen Zeitkonzept distanzieren sich die Witwen somit deutlich vom Patriarchat und ersetzen das damit verbundene absolute Zeitkonzept. Neben der Tatsache, dass die Zeit rückwärts abläuft, sind folgende Charakteristika des neuen Konzepts auffallend: Die Begriffe ,Monat“ oder ,Jahr‘ gelten als bedeutungslos und werden durch ,Stufen“ und ,Treppen“ der Selbstverwirklichung (Cañón 2007, 224) ersetzt, doch ,unlike the intimidating ladders of success or fame established by men, these ladders would go down and down only“ (Cañón 2007, 224). Darüber hinaus wählen die Witwen für jede Stufe eine Tugend, die sie als erstrebenswert erachten oder in deren Ausübung sie sich verbessern möchten (Cañón 2007, 224). Das relationale Zeitkonzept ebnet den Weg für das neue Selbstverständnis der Gruppe als diverse Gesellschaft ohne wirtschaftliche oder kulturell konstruierte Unterschiede zwischen ihren Bewohner*innen. Von dem Tag an, an dem der neue Zeitbegriff eingeführt wird, werden die Frauen im Roman nicht mehr als ,Ehefrau von“, ,Tochter von' oder ,Witwe von' bezeichnet. Sie selbst identifizieren sich also nicht mehr über die Namen ihrer Ehemänner oder Väter. In einer patriarchalischen Gesellschaft können Namen und vor allem die Identitätszuschreibung als jeman- 
dem zugehörig als ein Zeichen von machistischem Besitzanspruch gewertet werden. Nur noch mit ihrem Vornamen angesprochen, werden die Dorfbewohner`innen nun nicht mehr ausgehend von der Nicht-Existenz der Patriarchen charakterisiert und passen sich damit auch sprachlich nicht mehr in das patriarchale Rollengefüge ein. Gemeinsam erinnern sich die Dorfbewohner`innen daran, „that the first verb their mothers had taught them wasn't to be but to belong; therefore belonging would always come before being“ (Cañón 2007, 170 [Hvg. i.O.]). In der entstehenden ,Paragesellschaft' von New Mariquita erfahren sie zum ersten Mal die Bedeutung von individueller Selbstbestimmung und lernen den Wert ihres eigenen Daseins zu schätzen.

Die Dorfbewohner*innen kommen ohne ihre Männer gut zurecht, und so erweist sich die Konfrontation der neu gegründeten Gemeinschaft mit der Rückkehr einiger Männer des Dorfes, die erfolgreich aus einem Guerillalager geflohen sind, als umso herausfordernder. Unter dem euphemistischen Titel „The Men Who Asked for a Second Chance“ (Cañón 2007, 309) wird das Aufeinandertreffen von Rückkehrern und der diversen Gemeinschaft im letzten Romankapitel beschrieben. Die Männer fragen zunächst die Dorfbewohner*innen nach dem Weg nach Mariquita (Cañón 2007, 312) und trauen dann kaum ihren Augen, als ihnen bewusst wird, dass sie ihr Ziel bereits erreicht haben. Die Männer versuchen sofort, den patriarchalischen Status quo ante wieder herzustellen, wobei sie die Dorfbewohner^innen immer noch nicht als vollwertig und ihnen ebenbürtig begreifen. Als Vertreter der ,Mehrheitsgesellschaft' fassen sie die parallele Gemeinschaft von New Mariquita als Bedrohung auf und sehen in ihr, was Hiergeist als „ein Signum [...] der Auflösung bewährter Traditionen oder des Verlusts der eigenen Privilegien und Herrschaftsansprüche“ bezeichnet (2017, 11). Als die Dorfbewohner*innen über das Schicksal der Heimkehrer beraten, lassen die Patriarchen mit emotionalen Ausrufen wie „They're living like savages. We've a lot of work to do if we want to make this town livable“ (Cañón 2007, 318), „We're the only male survivors of this damned village. That's who we are! Mariquita belongs to us, and we've got to take charge again“ (Cañón 2007, 319) oder „No Woman’s going to tell me what to do [...]. [I want to] live in a place where women respect and obey men“ (Cañón 2007, 319) ihrer Wut und ihrem Unverständnis freien Lauf. Ihre stereotypen Wertungen deuten darauf hin, dass sie in ihrem voreingenommenen und diskriminierenden ideologischen Denken nicht dazu bereit sind, die neu gewonnene Identität und Unabhängigkeit der diversen Bewohner`innen der ,Paragesellschaft' ${ }^{\star} \mathrm{u}$ akzeptieren. Sie sind nach wie vor von der längst überholten patriarchalischen Norm lateinamerikanischer Kulturen überzeugt, die Barbara Seitz wie folgt definiert: „Whatever her social class, the ideal woman, according to tradition, occupies a supportive role in her relationships with men; her primary or focal area of activity is the home; she is sensuous, loving, and sub- 
missive“ (1991, 23). Nach einer gemeinschaftlichen Beratung konfrontieren die Dorfbewohner*innen die Rückkehrer mit der neuen Wirklichkeit, die sie geschaffen haben: „You see, the village which you used to live in no longer exists. You're now in New Mariquita, an independent [...] community with [...] special social, cultural and economic characteristics, and close bonds with nature“ (Cañón 2007, 322). Sie kommen zu dem Schluss, dass sie nie wieder in Erwägung ziehen werden, neue Mitglieder in die Gemeinschaft aufzunehmen, wenn sie nicht sicher sind, ob diese sich ihren neu geschaffenen Gepflogenheiten, Idealen und Regeln anpassen (Cañón 2007, 323).

\section{Die diverse, nachhaltige Gesellschaft von New Mariquita als ,Paragesellschaft`}

So ungewöhnlich der Beginn der Romanhandlung auf den ersten Blick erscheinen mag, er reflektiert doch die Alltagsrealität eines lateinamerikanischen Landes, das sich inmitten eines Krieges zwischen seinen Streitkräften, zersplitterten linksorientierten Guerillagruppen und rechtsorientierten paramilitärischen Verbänden befindet. Da der Staatsaufbau Kolumbiens nach den Unabhängigkeitskriegen Anfang des 19. Jahrhunderts lange Zeit scheiterte, wird es seit jeher als ,schwacher Staat“ kategorisiert und nimmt daher in Lateinamerika eine Sonderrolle ein (Hofmann und Nerb 2007, 209-210). Mit Beginn der grausamen ,la violencia'-Periode durch die Ermordung des Liberalen Jorge Eliécer Gaitán 1948 in Bogotá wird Gewalt eine wirksame Methode zur Durchsetzung politischer Interessen (Osterling 1989, 261). Der politisch motivierte Mord setzt eine tödliche Spirale der Gewalt in Gang, die sich bis ins einundzwanzigste Jahrhundert hinein steigert und erst seit wenigen Jahren von der kolumbianischen Regierung eingedämmt wird (Hofmann und Nerb 2007, 111).

Der zeitliche Ausgangspunkt der fiktionalen Erzählung ist ein Tag im November 1992. Er fällt damit in eine Zeit, in der die Kolumbianer*innen sowohl in städtischen als auch in ländlichen Gebieten noch häufig mit gewaltsamen Übergriffen, Entführungen und dem plötzlichen Verschwinden von Familienmitgliedern, Nachbarn und Bekannten konfrontiert waren. ${ }^{8}$ In diesem Teufelskreis der Gewalt entwickeln diametral entgegengesetzte sozioökonomische Interessen

8 Laut offizieller Berichterstattung hat Kolumbien 1985 eine Bevölkerung von etwa 26,5 Millionen Bürgern. Für das Jahr 1986 werden - Tod aufgrund von Straßenkriminalität mit eingeschlossen - 11.000 Gewalttodesfälle gezählt (Osterling 1989, 265-266), es wird jedoch davon ausgegangen, dass die Dunkelziffer hier weit höher liegt. 
von Landbesitzern und Bauern eine unkontrollierbare Dynamik, die zur Bildung von lokalen und regionalen Selbstverteidigungseinheiten führt, was wiederum die Gründung mehrerer linksgerichteter Guerillagruppen zur Folge hat (Hofmann und Nerb 2007, 111). ${ }^{9}$ Diese Gruppierungen, die unterschiedliche Ziele und Strategien verfolgen, fungieren als heterogene, stark fragmentierte Macht, ${ }^{10}$ welche ihre Interessen durchzusetzen versucht. Sie schrecken weder vor illegalem Drogenhandel, Entführungen und Erpressungen zurück, noch davor, gewaltsam mehr Personal für ihren rücksichtslosen Krieg zu rekrutieren. So lassen sie ganze Dörfer ohne männliche Bewohner zurück, was zum Entstehen sogenannter ,Witwenstädte، führt. Im Zuge ihres War on Drugs beginnen auch die Vereinigten Staaten seit den 1980er Jahren in Konfliktgebieten Lateinamerikas zu intervenieren. Als Reaktion auf linksgerichtete Guerillagruppen entstehen in den 1990er Jahren rechtsgerichtete paramilitärische Vereinigungen, die von Drogenkartellen finanziell unterstützt werden. Aufgrund der schwachen Stellung der kolumbianischen Streitkräfte (Korruption, kein staatliches Gewaltmonopol) können sich die zersplitterten Parteien in einem „undeclared internal war“ (Osterling 1989, 265 [Hvg. i.O.]) auf Mikroebene bekämpfen (Hofmann und Nerb 2007, 114-115 und 119-122). ${ }^{11}$

Die Entstehung von ,Paragesellschaften“ wird in als Krise wahrgenommenen Zeiten begünstigt, da sich „bisweilen beim Einzelnen oder bei kleineren oder größeren Teilen der Gesellschaft Zweifel daran [regen], ob die Staatsgewalt zur Einhaltung ihres Sicherheitsversprechens in der Lage und ob auf die Mitbürger de

\footnotetext{
9 Marxistische und prosozialistische Ideen beflügeln die bewaffnete Bauernbewegung. Diese Leitbilder werden in vielen Fällen von der Landbevölkerung übernommen (Hofmann und Nerb 2007, 112), was sich im Roman niederschlägt. Zum Beispiel scheinen die männlichen Bewohner kommunistische Versammlungen abzuhalten, von denen Mariquitas Bewohner*innen ausgeschlossen sind.

10 Die beiden größten Kräfte sind die FARC, die sich für soziale Gerechtigkeit und die Umverteilung von Eigentum einsetzt, und die ELN, die vor allem gegen multinationale Konzerne operiert (Hofmann und Nerb 2007, 113). Für weitere Informationen zu den beiden Gruppen siehe Osterling 1989, 292-300 (FARC) und 307-313 (ELN).

11 Diese gewaltreichen und scheinbar sinnlosen Kämpfe werden auch im Roman dargestellt. Jedes Kapitel endet mit einem kurzen Exkurs, in dem erzählt wird, was mit Mariquitas Männern, die im November 1992 entführt wurden, und mit anderen geschieht, die den verfeindeten Parteien angehören. Die Passagen reflektieren unter anderem das Leben von Kindersoldaten, die - nachdem ihre Eltern entführt oder ermordet wurden - keinen anderen Ausweg sehen, als sich dem Krieg anzuschließen (Cañón 2007, 17-22). Sie zeigen Grausamkeiten wie das Herausschneiden der Zungen bei überfallenen indigenen Völkern durch Guerillakräfte (Cañón 2007, 212-213) oder sie vermitteln die Gedanken eines abgehärteten kolumbianischen Soldaten, der erzählt, dass er „learned in the army [...] that the less contact you have with your victim, the easier it is to kill him“ (Cañón 2007, 65).
} 
facto Verlass ist“ (Biersack et al. 2019, 6 [Hvg. i.O.]). Die im Roman durch eine politische Krise (Überfall auf und Entführung von friedlichen Zivilisten im kolumbianischen Bürgerkrieg) verursachte, davon entkoppelte zweite Krise kann durch individuelle und kollektive Identitätsfindung überwunden werden. Nachdem die Dorfbewohner*innen mehrere Monate der Hungersnot und Dürre (Cañón 2007, 25) auf sich allein gestellt durchgestanden haben, verlassen sie sich weiterhin zunächst auf vertraute Orientierungsstiftungen wie den ihnen bekannten katholischen Glauben und bitten „the Lord to send us a truck full of men“ (Cañón 2007, 43). Bald jedoch ändern sie ihre Sichtweise und verfolgen das Ziel, das ehemalige Mariquita in ein Dorf „much better [...] than the men could have ever created“ (Cañón 2007, 30) zu verwandeln. Da sie sich im Laufe der Zeit nicht mehr an die Gesichter ihrer Ehemänner, Brüder oder Freunde erinnern können (Cañón 2007, 93) und sie zugeben müssen, dass „their men died in their hearts“ (Cañón 2007, 101), stellen die Dorfbewohner`innen schließlich fest, dass „Mariquita’s problem is not the lack of men but the lack of resources,, (Cañonn 2007, 101). Diese Erkenntnis ermöglicht ihnen, die krisengebeutelte und zerrüttete Dorfgemeinschaft zu einer nach ihren Wertevorstellungen funktionierenden ,Paragesellschaft‘ umzustrukturieren. Mit der Wahl eines nicht genauer spezifizierten Dorfes im Kolumbien der 1990er Jahre als Schauplatz der Erzählung wertet der Text die negativ konnotierte Situation um, indem er den Marginalisierten eine Stimme verleiht und die bestehenden Hierarchien auf fiktionaler Ebene invertiert. Darüber hinaus hebt die natürliche Barriere, welche das Dorf von der Außenwelt abschirmt, symbolisch den Kontrast zwischen den beiden ,Welten“ hervor: der patriarchalisch geprägten ,Mehrheitsgesellschaft', die als negativ und sogar bedrohlich empfunden wird, sowie der diversen ,Paragesellschaft', die als positive, funktionierende und nachhaltige Alternative präsentiert wird.

Eine weiterer Aspekt, der aufzeigt, dass Tales from the Town of Widows Gesellschaft neu denkt und es vermag, sich auf fiktionaler Ebene fruchtbar mit gesellschaftlich virulenten Problemen auseinanderzusetzen, ist die im Roman dargestellte Überwindung etablierter, aber nicht mehr vertrauenswürdiger Institutionen. Wie Martin Biersack et al. in der Einleitung zum Band Parallelgesellschaften. Instrumentalisierungen und Inszenierungen in Politik, Kultur und Literatur formulieren, wird das Entstehen einer ,Paragesellschaft' dadurch begünstigt, dass sich gesellschaftlich etablierte Institutionen in einer Vertrauenskrise befinden $(2019$, 9). Im Roman werden die Struktur des Patriarchats und die damit verbundene Institution der katholischen Kirche als nicht länger vertrauenswürdig dargestellt. Das Vertrauen der Dorfbewohnerinnen in die Patriarchen, in den katholischen Priester und in das absolute Zeitkonzept, welches - ebenso wie die Ausübung des katholischen Glaubens - als eine ideologisch und gesellschaftlich konstruierte Fessel erscheint, die nun endlich 
gelöst werden kann, schwindet. Lösungsstrategien für das Überwinden der Krise, die den sozialen und gesellschaftlichen Ansatz der ,Mehrheitsgesellschaft‘ unterstützen, werden nach unerfreulichen Zwischenfällen wie den Fortpflanzungskampagnen nicht mehr angenommen. Da die Ablösung vom Patriarchat schrittweise und nicht plötzlich erfolgt, beleuchtet die Romanhandlung besonders deutlich, welche Strukturen als überholt gelten und somit einen klaren Unterschied zur sich im Aufbau befindenden ,Paragesellschaft‘ darstellen. Neben dem ethisch korrumpierten Priester als Vertreter des katholischen Glaubens ist auch das absolute Zeitkonzept in den Wertevorstellungen der diversen ,Paragesellschaft‘ nicht mehr akzeptabel. Ein wichtiges Merkmal des neuen Konzepts ist, dass die Zeit in New Mariquita rückwärts fließt. Die naheliegende Idee dahinter vermag Leserinnen zu überraschen: „[W]hen time moves backward, people have a chance to change the course of their lives!“ Mit diesem Konzept, so die Dorfbewohnerinnen, „[w]e’ll go back in time, fix the many problems there are in our history, and create a prosperous future for all of us“ (Cañón 2007, 221). Was anfänglich etwas naiv anmuten mag, reift zur Überzeugung heran, dass sich eine diverse Gesellschaft, in der sozial geschaffene, auf Genderdifferenz beruhende Ungerechtigkeiten abgeschwächt werden, durchaus entfalten kann. Weiterhin kann das Einsetzen des neuen Zeitkonzepts auch als Kritik am linearen Fortschrittsgedanken gelesen werden. Die Dorfbewohner*innen kehren der von der ,Mehrheitsgesellschaft‘ angenommenen Progressivität, die nur eindimensional verstanden wird und die Geschichte(n) von benachteiligten Minderheiten wie Frauen oder LGBTQ marginalisiert oder überschreibt, aktiv den Rücken. Indem sie das absolute Zeitkonzept umkehren und für ihr Zusammenleben ein neues konzipieren, in dem die Zeit rückwärts läuft, spalten sie sich auch in diesem Punkt auf fast reaktionäre Weise von der ,Mehrheitsgesellschaft‘ ab. Die nachvollziehbare Haltung der fiktiven Charaktere unterstreicht das Potential der literarischen Utopie und der in ihr dargestellten imaginären ,Paragesellschaft‘. Indem die Erzählung aufzeigt, dass ein kleiner Zwischenfall nämlich die Tatsache, dass die Kirchturmuhr nicht mehr funktioniert - die politische Stoßrichtung und den gesellschaftlichen Fokus einer Gesellschaft verändern kann, veranlasst sie Leser`innen, eigene soziale Missstände zu reflektieren und darüber nachzudenken, wie diese möglicherweise überwunden werden könnten.

Nachdem sie verschiedene Modelle des Zusammenlebens erprobt und nach und nach gesellschaftliche Positionen wie die der Bürgermeisterin, der Polizeibeamtin oder der Richterin als Überbleibsel eines patriarchalischen und von Männern dominierten Mariquita abgeschafft haben, finden die Dorfbewohner*innen die für sie am besten funktionierende, sozialistisch-autarke Form eines friedlichen und diversen Konvivialismus. Tales from the Town of Widows verhandelt somit literarisch, was Biersack et al. als dynamische Gesellschaft bezeichnen, die 
keine ontologische Entität darstellt, sondern vielmehr stets erarbeitet wird (2019, 12). Dabei unterstreicht der utopische Roman den Gedanken - und macht somit auch Leser`innen deutlich -, dass gesellschaftliche Veränderung ein möglicher, aber langanhaltender Prozess ist, der beständig ausgehandelt werden muss. Als präsentischer Einschub in die eigentliche Erzählung im epischen Präteritum erklärt der heterodiegetische Erzähler, wie die Alternative zur ,Mehrheitsgesellschaft' organisiert ist: $^{12}$

The community of New Mariquita has no chief or council. Major decisions are reached by consensus, in a participatory, inclusive decision-making process that allows all ninetythree residents to have a voice. The smaller, sun-to-sun decisions are made by the caretaker of each particular area. For instance, every house has a meal caretaker and a helper. They cook all three meals and make sure their housemates get all the food they need. Supplies of food for each kitchen are equally distributed by the store caretaker, who also threshes or husks grain, dries any surplus of meat and fish, and stores all sorts of food in large jars made of clay. In a like manner farm products are collected and brought to the store by the farm caretaker. She oversees the communal farm, the planting and harvesting of crops, and, with input from the community, decides what produce and animals need to be raised. Every caretaker position, every task and small chore, is rotated among the villagers on a rungly ${ }^{13}$ basis. Wool and cotton are allotted to old women, who are charged with the task of spinning and weaving. Everyone acts on her own, but if a woman (or Santiago Marín) has a problem, she is encouraged to bring the issue to the community consensus process.

(Cañón 2007, 321)

Vor allem die starken sozialen Bindungen, die auch homosexuelle Beziehungen einschließen, sowie der dynamische und flexible Prozess der Gründung der nachhaltigen, diversen und autarken Gemeinschaft geben den Dorfbewohner*innen bei Rückschlägen Halt. Befähigt durch ein starkes Gemeinschaftsgefühl und durch die Erkenntnis, dass sie das Recht haben, ihre eigenen Entscheidungen zu treffen (Cañón 2007, 159), finden die einzelnen Dorfbewohner^innen ihre Stimme und sind so in der Lage, ihre individuelle und kollektive Meinung zu artikulieren. Selbst als die Rückkehrer unter Androhung von Gewalt nicht akzeptieren wollen, dass in der alternativen Gesellschaft andere Regeln herrschen, stehen die Dorfbewohner*innen zusammen und vertreiben diejenigen, die ihre neu gewonnene Freiheit und Identität untergraben möchten. Im Allgemeinen kann die Ankunft

12 Die Verwendung verschiedener Zeitformen stellt den fiktionalen Status des Romans auf die Probe, da sie nicht nur auf den Leseprozess anspielen, sondern darüber hinaus suggerieren, dass eine ,Paragesellschaft‘ wie New Mariquita tatsächlich existieren könnte.

13 Wie beschrieben, werden einige Begriffe des Zeitkonzepts der ,Mehrheitsgesellschaft" im neuen Zeitkonzept des Dorfes durch andere ersetzt. Es gibt nun statt Monaten und Jahren „,rungs‘ and ,ladders‘ to self-improvement“ (Cañón 2007, 224). Der Neologismus ,rungly‘ leitet sich somit von rungs ab. 
der rückkehrenden Patriarchen als ein zweischneidiges Schwert betrachtet werden. Einerseits stellen die Männer mit ihrer überholten patriarchalischen Haltung und Denkweise eine Bedrohung für die diverse ,Paragesellschaft' dar. Auf der anderen Seite kann ihre Rückkehr auch als Chance gewertet werden, da sich die Dorfbewohner*innen in den Jahren ihrer Abwesenheit nicht fortpflanzen konnten. Das Ultimatum und die Bedingungen, die die Dorfbewohner`innen stellen, können als Antwortmöglichkeit auf die kulturelle und soziale Situation in Kolumbien gewertet werden, die Frauen und LGBTQ zum Schweigen bringt, sie unsichtbar macht, sie diskriminiert, sie verletzt und ihnen passive Rollen und somit keine eigenen Identitäten zuschreibt. New Mariquitas Bewohner^innen zufolge ist „[e]quality between individuals and between the sexes [...] number one“ (Cañón 2007, 332), weshalb sie einsichtigen Rückkehrern eine zweite Chance geben und ihnen ermöglichen, mit ihnen nach den Regeln der diversen und nachhaltigen ,Paragesellschaft‘ $\mathrm{zu}$ leben.

\section{Abschließende Worte}

Elaine Showalter zufolge ist der Wunsch nach einer klaren Definition von Gender in Zeiten von kultureller und politischer Unsicherheit und Krise besonders groß (1990, 4). Tales from the Town of Widows befasst sich mit einer solchen Periode hoher kultureller Unsicherheit, die auf gesellschaftlich tradierten Genderoppositionen beruht und zusätzlich auch aus den Grausamkeiten des Bürgerkriegs in Kolumbien resultiert. Eines der Schreckensszenarien dieses Krieges ist sicherlich das plötzliche Auftauchen von Witwenstädten, in denen nach einem Überfall durch kommunistische Guerilla-Gruppen oder paramilitärische Kräfte und der Entführung oder Ermordung der männlichen Bewohner meist nur Bewohner^innen zurückgelassen werden. James Cañóns fiktionaler Vorschlag für ein produktives, diverses Miteinander eröffnet einen Dialog, in dem binäre patriarchalische Oppositionsstrukturen durchbrochen werden. Mit seiner „ability to encapsulate epic political history into poignant poetic prose“ (Uchmanowicz 2006) macht er auf die problematische gesellschaftliche Wirklichkeit von kolumbianischen Frauen und LGBTQ aufmerksam, die unabhängig von ihrem sozialen Status marginalisiert und diskriminiert werden. In ihrer die patriarchalischen Grenzen überschreitenden Sehnsucht nach einer klaren Definition von Gender schließen die Dorfbewohner^innen das im Patriarchat stigmatisierte ,Andere“ niemals aus. Im Gegenteil: In New Mariquita werden weder Gender- noch etwaige kulturelle Differenzen als Hindernis gesehen, da in der neu geschaffenen ,Paragesellschaft nicht Divergenz, sondern Diversität und Heterogenität großgeschrieben werden 
und das nicht-anthropozentrische Verhältnis zwischen Mensch und Natur in den Vordergrund gestellt wird. Indem er sich gesellschaftskritisch gegenüber der gegenwärtigen kulturellen und patriarchalischen Starrheit positioniert (Tangney 2001, 44) und von der ,Mehrheitsgesellschaft' ungenutzte Potenziale ausschöpft, stößt der Roman ein Umdenken sowie eine (Neu-)Imagination anderer, funktionierender Gesellschaftsformen an und trägt somit aktiv dazu bei, Gesellschaft neu zu denken. Dabei führt er Leser`innen vor Augen, dass Gesellschaft keineswegs ein ontologisches, nicht veränderbares Konstrukt ist, sondern dass ein dynamischer, von Rückschlägen geprägter Entwurf neuer Wertvorstellungen und Verhaltensweisen für soziale Transformation entscheidend ist.

\section{Bibliographie}

Aubès, Françoise. „Si loin, si près: Diaspora et globalisation chez les écrivains transnationaux“. América: Cahiers du CRICCAL 39 (2010): 23-29.

Biersack, Martin, Teresa Hiergeist und Benjamin Loy. „Das Leben der Anderen. Historische, soziologische und narrative Dimensionen paralleler Sozialität“. Parallelgesellschaften. Instrumentalisierungen und Inszenierungen in Politik, Kultur und Literatur (Romanische Studien, Beihefte 8). Hg. Martin Biersack, Teresa Hiergeist und Benjamin Loy. München: AVM, 2019. 5-17.

Cañón, James. Tales from the Town of Widows. London: Harper Perennial, 2007.

Cañón, James. „Mariquita“. The Novelist's Lexicon. Writers on the Words That Define Their Work. Hg. Villa Gillet. New York: Columbia University Press, 2011. 65-66.

Hiergeist, Teresa. „Selbst, anders, neu. Reflexionen zu den kulturellen und ästhetischen Bedeutungen von ,Parallel- und Alternativgesellschaften'“. Parallel- und Alternativgesellschaften in den Gegenwartsliteraturen. Hg. Teresa Hiergeist. Würzburg: Königshausen \& Neumann, 2017. 7-24.

Hofmann, Aletta und Tobias Nerb. „Kolumbien: Zwischen Guerillakrieg, Drogenkartellen und Reststaatlichkeit“. Wenn Staaten scheitern. Theorie und Empirie des Staatenzerfalls. Hg. Alexander Straßner und Margarete Klein. Wiesbaden: VS, 2007. 109-130.

Klarer, Mario. Frau und Utopie: feministische Literaturtheorie und utopischer Diskurs im angloamerikanischen Roman. Darmstadt: Wissenschaftliche Buchgesellschaft, 1993.

Layh, Susanna. Finstere neue Welten. Gattungsparadigmatische Transformationen der literarischen Utopie und Dystopie. Würzburg: Königshausen \& Neumann, 2014.

m.A. Convivialist Manifesto. A Declaration of Interdependence (Global Dialogues 3). Duisburg: Käte Hamburger Kolleg/Centre for Global Cooperation Research (KHK/GCR21), 2014.

McDowell, Lila Cole. The Utopian Impulse in Chilean and Mexican Novels 1990-2005. University of California: Dissertation, 2008.

Osterling, Jorge P. Democracy in Colombia. Clientelist Politics and Guerrilla Warfare. New Brunswick: Transaction Publishers, 1989.

Pfaelzer, Jean. The Utopian Novel in America 1886-1896: The Politics of Form. Pittsburgh: University of Pittsburgh Press, 1984. 
Quiñones Mayo, Yolanda und Rosa Perla Resnick. „The Impact of Machismo on Hispanic Women“. AFFILIA 11.3 (1996): 257-277.

Relf, Jan. „Women in Retreat: The Politics of Separatism in Women's Literary Utopias“. Utopian Studies 2.1/2 (1991): 131-146.

Romaine, Suzanne. Communicating Gender. London: Routledge, 1999.

Schölderle, Thomas. Utopia und Utopie. Thomas Morus, die Geschichte der Utopie und die Kontroverse um ihren Begriff. Baden-Baden: Nomos, 2011.

Seitz, Barbara. „Songs, Identity, and Women's Liberation in Nicaragua“. Latin American Music Review/Revista de Música Latinoamericana 12.1 (1991): 21-41.

Showalter, Elaine. Sexual Anarchy: Gender and Culture at the Fin de Siècle. New York: Viking, 1990.

Tangney, ShaunAnne. „Brave New Girls: Female Archetypes, Border Crashing, and Utopia in Kate Braverman's Palm Latitudes“. Rocky Mountain Review of Language and Literature 55.1 (2001): 43-61.

Uchmanowicz, Pauline. „Book Review: Tales from the Town of Widows“. Chronogram. 27. Dezember 2006. https://www.chronogram.com/hudsonvalley/book-review-talesfrom-the-town-of-widows-and-chronicles-from-the-land-of-men/Content?oid=2171924 (20. August 2020).

Werder, Peter R. Utopien der Gegenwart. Zwischen Tradition, Fokussierung und Virtualität. Zürich: Seismo, 2009. 